\title{
INSPEÇÃO DA EQUAÇÃO DE BLAKE-KOZENY-CARMAN NA FLUIDIZAÇÃO EM POROSIDADES ELEVADAS: AVALIAÇÃO DO FATOR $\Omega$ E DA CONSTANTE DE BURKE-PLUMMER
}

\author{
M. F. P. MOREIRA ${ }^{1 *}$, J. T. FREIRE ${ }^{2}$ \\ ${ }^{1}$ Universidade Estadual do Oeste do Paraná, Departamento de Engenharia Química \\ ${ }^{2}$ Universidade Federal de São Carlos, Departamento de Engenharia Química \\ *e-mail: marcosfpmoreira@gmail.com
}

\begin{abstract}
RESUMO
O objetivo deste trabalho foi avaliar a equação de Blake-Kozeny-Carman à luz da fluidodinâmica da partícula isolada a fim de determinar teoricamente as influências da porosidade e do número de Reynolds sobre o fator $\Omega$ e sobre a constante de Burke-Plummer. Verificou-se que tanto o fator $\Omega$ como a constante de Burke-Plummer diminuem acentuadamente com o aumento do número de Reynolds da partícula e também com o aumento da porosidade, tendência teórica essa confirmada no confronto com resultados experimentais. Foram propostas equações para a constante de Burke-Plummer em uma ampla faixa de número de Reynolds da partícula, que se estende desde o regime de Stokes até o regime turbulento.
\end{abstract}

\section{INTRODUÇÃO}

A determinação do comportamento de um fluido ao percolar um determinado meio poroso é assunto de interesse da humanidade há muitos séculos, seja pela necessidade de se saber a energia a ser imposta ao fluido ou a determinação do tempo necessário para que tal processo ocorra. $\mathrm{O}$ escoamento em meios porosos está presente em incontáveis situações como na irrigação da terra para o plantio, na aeração de uma massa de grãos, nos processos de separação por membranas, entre tantos outros.

Uma situação particular de meio poroso são os meios porosos expandidos, mais especificamente os leitos fluidizados, responsáveis por altas taxas de transferências de calor e de massa nos processos de engenharia. A compreensão da fluidodinâmica dos leitos fluidizados pode se dar através de (Barnea e Mizrahi, 1973):
- modelos teóricos (Equações de NavierStokes);

- modelos semi-empíricos (analogia entre a força de arraste sobre partículas esféricas em suspensão e a queda de pressão em leitos fixos, analogia entre as equações de NavierStokes e a Lei de Darcy, analogia entre o aumento da viscosidade aparente da solução e a quantidade de movimento), e de

- modelos empíricos.

Dentro dos modelos semi-empíricos, a analogia entre a força de arraste sobre partículas esféricas em suspensão e a queda de pressão em leitos fixos aparece como um dos modelos mais utilizados na literatura.

A queda de pressão em leitos fixos pode ser obtida a partir da Equação de Ergun (Ergun, 1952) para leitos granulares ou pelo modelo de Comiti e Renaud (1989), ambos baseados na teoria capilar. 
A Equação de Ergun (Ergun, 1952) foi desenvolvida a partir das equações de BlakeKozeny e Burke-Plummer (Plessis e Woudberg, 2008) aplicada a esferas. Macdonald et al. (1979) propuseram a inclusão da esfericidade da partícula na equação de Ergun, mas apesar disso, a equação é restrita a condições onde o efeito de parede é desprezível e a porosidades na faixa de 0,35 a 0,5 . É possível ainda modificar a equação de Ergun para a inclusão do efeito de parede (Eisfeld e Schnitzlein, 2001; Ribeiro et al., 2010). Puncochar e Drahos (2000) realizaram um estudo a respeito da aplicabilidade dos modelos capilares para a determinação da queda de pressão tendo como foco principal a equação de Ergun.

Em relação à influência da porosidade, para porosidades acima de 0,5 os valores constantes presentes na equação de Ergun, 150 (constante de Blake-Kozeny-Carman) e 1,75 (constante de Burke-Plummer), tornamse funções da porosidade como pode ser verificado na literatura (Massarani, 1989; Nemec e Levec, 2005; Deen et al., 2007; Plessis e Woudberg, 2008). Dessa forma, a utilização da equação de Blake-KozenyCarman é indicada em condições de porosidade onde a equação de Ergun não se aplica, mas com o inconveniente de se obter tais constantes como funções da porosidade (Zhu et al., 1995).

Para elevadas porosidades, tanto o comportamento do fator $\Omega$ como o da constante de Burke-Plummer ainda carecem de informações. Em vista disso, o objetivo deste trabalho é avaliar teoricamente o fator $\Omega$ e a constante de Burke-Plummer presentes na equação de Blake-Kozeny-Carman estudando as influências do número do Reynolds e da porosidade em condições de elevadas porosidades à luz da dinâmica da partícula isolada.

\section{A EQUAÇÃO BASE DO ESTUDO}

\subsection{O Modelo Capilar, o Fator $\Omega$ e a Constante de Burke-Plummer}

A queda de pressão em meios porosos é dada basicamente pela equação ou "Lei" de Darcy (Dacy, 1856):

$-\frac{\Delta \mathrm{P}}{\mathrm{L}}=\frac{\mu}{\mathrm{k}} \mathrm{q}$

onde $\mathrm{P}$ representa a pressão piezométrica, $\mathrm{k}$ e L são a permeabilidade e o comprimento do meio poroso respectivamente, $\mu$ é a viscosidade dinâmica do fluido e q é a velocidade superficial do fluido. Considerando uma analogia do escoamento darcyano em meio poroso com o escoamento laminar em dutos paralelos, similares e retilíneos, onde

$-\frac{\Delta \mathrm{P}}{\mathrm{L}}=\frac{\beta}{\mathrm{R}_{\mathrm{h}}^{2}} \mu . \mathrm{U}$

sendo $R_{h}$ o raio hidráulico, " $u$ " a velocidade do fluido e $\beta$ um fator adimensional que depende da forma da seção transversal do duto, chega-se a (Carman, 1937):

$-\frac{\Delta \mathrm{P}}{\mathrm{L}}=\frac{\mathrm{k}_{\mathrm{koz}} \mathrm{S}_{\mathrm{M}}^{2}}{\varepsilon^{3}} \mu \mathrm{q}$

onde

$\mathrm{R}_{\mathrm{h}}=\frac{\varepsilon}{\mathrm{S}_{\mathrm{M}}}$

$\beta=\mathrm{k}_{\text {ког }}$

$\mathrm{u}=\frac{\mathrm{q}}{\varepsilon}$ 
onde $\mathrm{k}_{\mathrm{KOZ}}$ é a constante de Kozeny dada por (Carman, 1937):

$\mathrm{k}_{\text {ког }}=\mathrm{k}_{0}\left(\frac{\mathrm{L}_{\mathrm{E}}}{\mathrm{L}}\right)^{2}$

onde $\mathrm{L}_{\mathrm{E}}$ é o comprimento tortuoso, $\mathrm{L}$ é o comprimento sem tortuosidade e $\mathrm{k}_{0}$ é uma constante que depende da seção transversal do canal (Carman, 1937) sendo igual a 2 para canais circulares. Para esferas a razão $\mathrm{L}_{\mathrm{E}} / \mathrm{L}$ equivale a $\pi / 2$. " $\mathrm{S}_{M}$ " é a superfície específica molhada do meio poroso e " $\varepsilon$ " é a porosidade.

Considerando que a área de todas as partículas esteja em contato com o fluido (fluidização), então

$\mathrm{S}_{\mathrm{M}}=(1-\varepsilon) \frac{\mathrm{A}_{\mathrm{p}}}{\mathrm{V}_{\mathrm{p}}}=\frac{6(1-\varepsilon)}{\phi \cdot \mathrm{dp}}$

onde $A_{p}$ e $V_{p}$ são a área e o volume da partícula, $\phi$ a sua esfericidade e dp o diâmetro equivalente. Substituindo a Equação 8 na Equação 3 tem-se que:

$-\frac{\Delta \mathrm{P}}{\mathrm{L}}=36 \mathrm{k}_{\text {ког }} \frac{(1-\varepsilon)^{2}}{(\phi \cdot \mathrm{dp})^{2} \varepsilon^{3}} \mu \mathrm{q}$

com a permeabilidade da Equação 1 sendo dada por:

$$
\mathrm{k}=\frac{(\phi \cdot \mathrm{dp})^{2} \varepsilon^{3}}{36 \mathrm{k}_{\text {ког }}(1-\varepsilon)^{2}}
$$

Da analogia das equações que descrevem o escoamento a altas vazões no meio poroso e no duto retilíneo, dadas por (Massarani, 2002):

$$
-\frac{\Delta \mathrm{P}}{\mathrm{L}}=\frac{\mathrm{c} \cdot \rho \cdot \mathrm{q}^{2}}{\sqrt{\mathrm{k}}}
$$

$-\frac{\Delta \mathrm{P}}{\mathrm{L}}=\frac{\mathrm{f} \cdot \rho \cdot \mathrm{u}^{2}}{2 \cdot \mathrm{R}_{\mathrm{h}}}$

sendo a permeabilidade dada pela Equação 10 e o $R_{h}$ pela Equação 4, obtém-se

$$
\mathrm{c}=\frac{\Omega}{\varepsilon^{3 / 2}}
$$

e

$$
-\frac{\Delta \mathrm{P}}{\mathrm{L}}=\Omega \sqrt{36 \mathrm{k}_{\mathrm{коz}}} \frac{(1-\varepsilon)}{(\phi \cdot \mathrm{dp}) \varepsilon^{3}} \rho \cdot \mathrm{q}^{2}
$$

A união das Equações 9 e 11, fazendo (Nemec e Levec, 2005):

$$
\mathrm{A}=36 \mathrm{k}_{\text {ког }}
$$

onde "A" é a constante de Blake-KozenyCarman e fazendo (Nemec e Levec, 2005):

$\mathrm{B}=\Omega \sqrt{36 \mathrm{k}_{\text {ког }}}$

onde "B" é a constante de Burke-Plummer, leva a (Macdonald et al., 1979):

$$
-\frac{\Delta \mathrm{P}}{\mathrm{L}}=\mathrm{A} \frac{(1-\varepsilon)^{2}}{(\phi \cdot \mathrm{dp})^{2} \varepsilon^{3}} \mu \mathrm{q}+\mathrm{B} \frac{(1-\varepsilon)}{(\phi \cdot \mathrm{dp}) \varepsilon^{3}} \rho \cdot \mathrm{q}^{2}
$$

que é a Equação de Ergun modificada, onde os valores de A e B propostos originalmente por Ergun (1952) são constantes e iguais a 150 e 1,75 respectivamente. Quando A e B são dados pelas Equações 15 e 16, a Equação 17 é conhecida como a Equação de BlakeKozeny-Carman.

\subsection{Analogia entre o Modelo Capilar e a Dinâmica da Partícula Isolada}

Sabe-se da dinâmica da partícula durante a fluidização que: 


$$
(1-\varepsilon)\left(\rho_{s}-\rho\right) g=\mu . \varphi_{1} \cdot \mathrm{U}+\rho \cdot \varphi_{2} \cdot \mathrm{U}^{2}
$$

onde

$\varphi_{1}=\frac{\varepsilon}{\mathrm{k}}$

$\varphi_{2}=\Omega \cdot \sqrt{\frac{\varepsilon}{\mathrm{k}}}$

$\Omega=\frac{\varphi_{2}}{\sqrt{\varphi_{1}}}$

Sabe-se ainda que o coeficiente de arraste $\left(C_{D}\right)$ é dado por:

$$
\mathrm{C}_{\mathrm{D}}=\frac{4}{3} \cdot \frac{\mathrm{d}_{\mathrm{p}}\left(\rho_{\mathrm{s}}-\rho\right) \cdot \mathrm{g}}{\rho \mathrm{v}^{2}}
$$

Substituindo a Equação 22 na Equação 18 tem-se que (Massarani, 2002):

$$
\frac{3}{4}(1-\varepsilon) \frac{\mu^{2}}{\rho \cdot \mathrm{d}_{\mathrm{p}}^{3}} \mathrm{C}_{\mathrm{D}} \cdot \mathrm{Re}^{2}=\mu \cdot \varphi_{1} \cdot \mathrm{U}+\rho \cdot \varphi_{2} \cdot \mathrm{U}^{2}
$$

ou ainda

$$
\mathrm{C}_{\mathrm{D}}=\frac{4}{3 \cdot \operatorname{Re}} \psi_{1} \cdot\left(\frac{\mathrm{U}}{\mathrm{V}_{\mathrm{o}}}\right)+\frac{4}{3} \psi_{2} \cdot\left(\frac{\mathrm{U}}{\mathrm{v}_{\mathrm{o}}}\right)^{2}
$$

onde

$$
\begin{aligned}
& \psi_{1}=\frac{\varepsilon \cdot \mathrm{d}_{\mathrm{r}}^{2}}{(1-\varepsilon) \cdot \mathrm{k}}=\frac{36 \mathrm{k}_{\mathrm{kog}} \cdot(1-\varepsilon)}{(\phi \cdot \varepsilon)^{2}}=\mathrm{A} \frac{(1-\varepsilon)}{(\phi \cdot \varepsilon)^{2}} \\
& \psi_{2}=\frac{\mathcal{E}^{2} \cdot c \cdot \mathrm{d}_{\mathrm{r}}}{(1-\varepsilon) \cdot \sqrt{\mathrm{k}}}=\frac{\sqrt{36 \mathrm{k}_{\mathrm{ko} \alpha}} \cdot \Omega}{\phi \cdot \varepsilon}=\frac{\mathrm{B}}{\phi \cdot \varepsilon}
\end{aligned}
$$

A razão $U / \mathrm{v}_{\infty}$ pode ser dada pela abordagem fundamental de Hawksley (Barnea e Mizrahi, 1973):

$$
\frac{\mathrm{U}}{\mathrm{V}_{\mathrm{o}}}=\varepsilon \cdot \frac{\mu}{\mu_{\mathrm{Ap}}}
$$

onde a razão de viscosidade do fluido e viscosidade aparente da solução para porosidades tendendo à unidade e partículas esféricas pode ser dada pela equação de Einstein (Roscoe, 1952):

$\frac{\mu_{A p}}{\mu}=3,5-2,5 . \varepsilon$

\subsection{Equação para Obtenção do Fator $\Omega$ e da Constante de Burke-Plummer}

A equação para obtenção do fator $\Omega$ foi gerada a partir da Equação 24 sendo a constante de Kozeny tomada como (Massarani e Santana, 1994):

$$
\mathrm{k}_{\mathrm{koz}}=\frac{\varepsilon^{2}}{2(1-\varepsilon)(4,8 \varepsilon-3,8)} \quad 0,9<\varepsilon<1
$$

a partícula considerada como sendo esfera $(\phi=1)$ e a razão $\mathrm{U} / \mathrm{v}_{\infty}$ dada pelas Equações 27 e 28. Dessa forma, obteve-se:

$$
\begin{aligned}
\mathrm{C}_{\mathrm{o}}=\frac{24 \varepsilon}{(4,8 \varepsilon-3,8)(3,5-2,5 \varepsilon) \cdot \operatorname{Re}}+ \\
\quad+\frac{8 \Omega}{\sqrt{2(1-\varepsilon)(4,8 \varepsilon-3,8)}} \cdot \frac{\varepsilon^{2}}{(3,5-2,5 \varepsilon)^{2}}
\end{aligned}
$$

Para o cálculo de $C_{D}$ é necessário o conhecimento do número de Reynolds (ou o regime de escoamento). Neste trabalho foram considerados os seguintes regimes referentes à fluidodinâmica da partícula isolada: 
a) regime de Stokes $(\operatorname{Re} \leq 0,1)$

b) regime de transição $(0,1<\operatorname{Re}<1.000)$,

c) regime de Newton $(1.000 \leq \mathrm{Re} \leq 10.000)$,

d) regime turbulento ( $\operatorname{Re} \geq 400.000)$

O coeficiente de arraste para o regime de Stokes foi considerado como (Barnea e Mizrahi, 1973):

$$
\mathrm{C}_{\mathrm{D}}=\frac{24}{\mathrm{Re}}
$$

Para o regime de transição, o coeficiente de arraste foi dado pela equação proposta por Massarani (2002), a qual se baseia nas assíntotas dos regimes de Stokes e de Newton:

$$
C_{D}=\left[\left(\frac{24}{\operatorname{Re}}\right)^{0.63}+0,43^{0.63}\right]^{10.63}
$$

O coeficiente de arraste para os regimes de Newton e turbulento foram considerados como constantes e iguais a 0,44 (Barnea e Mizrahi, 1973) e 0,2 (Foust et al., 1980) respectivamente.

A determinação da constante de BurkePlummer se deu a partir da Equação $16 \mathrm{em}$ posse dos valores do fator $\Omega$ obtidos da Equação 30 e dos valores da constante de Kozeny obtidos da Equação 29.

\section{RESULTADOS E DISCUSSÕES}

3.1 Comportamento do Fator $\Omega$ e da Constante de Burke-Plummer em função do Número de Reynolds

Verifica-se na Figura 1 que o fator $\Omega$ diminui com o aumento do número de Reynolds da partícula para todas as porosidades avaliadas até o final do regime de transição. No regime de Newton há um pequeno aumento do fator $\Omega$ com o aumento do número de Reynolds da partícula. Para $\Omega$ foram obtidos valores de 0,004 a 100 nas faixas de Reynolds e porosidade apresentadas na Figura 1.

A relação entre o logaritmo de $\Omega$ e o logaritmo do número de Reynolds da partícula é do tipo exponencial, ou seja:

$\log \Omega=$ a.exp$[-$ b. $\log (\operatorname{Re})]$

ou de forma mais simplificada

$\Omega=\frac{\mathrm{m}}{\operatorname{Re}^{\mathrm{N}}}$

Figura 1 - O fator $\Omega$ em função do número de Reynolds da partícula.

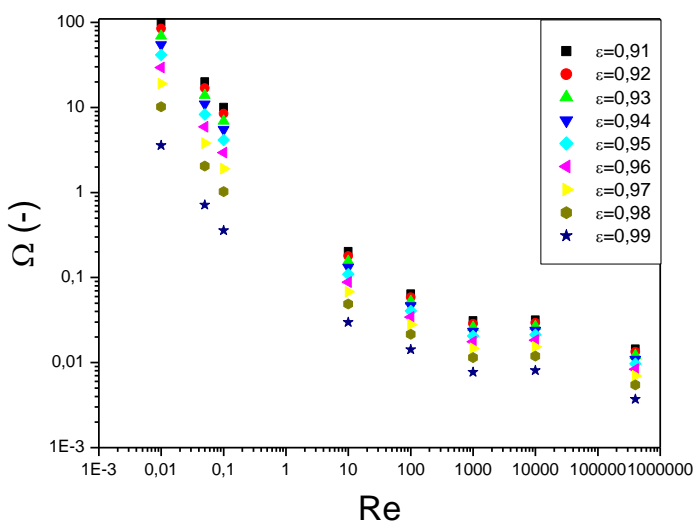

Fonte: Autor (2015).

A Figura 2 apresenta o comportamento do coeficiente de Burke-Plummer (B) em função do número de Reynolds da partícula parametrizado na porosidade.

Verificou-se um comportamento qualitativo de $\mathrm{B}$ em função do número de Reynolds e da porosidade semelhante ao comportamento apresentado pelo fator $\Omega$. Foram obtidos para B valores de 0,16 a 1.700 nas faixas de Reynolds e porosidade apresentadas na Figura 2. 
Figura 2 - Constante de Burke-Plummer (B) em função do número de Reynolds da partícula.

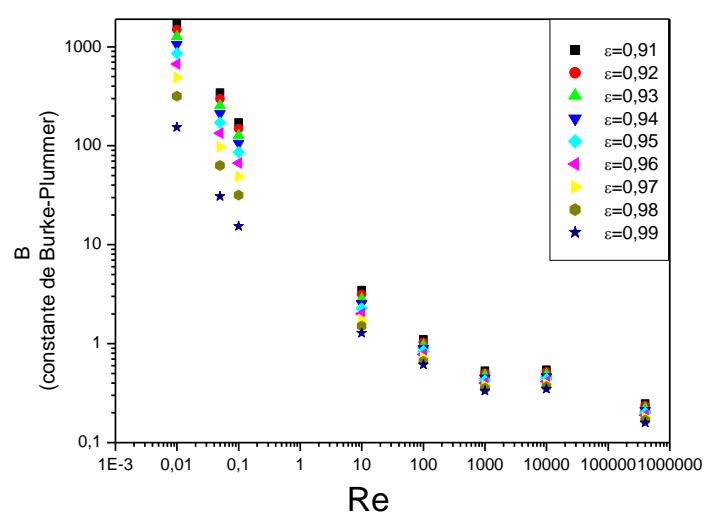

Fonte: Autor (2015).

3.2 Comportamento do Fator $\Omega$ e da Constante de Burke-Plummer em função da Porosidade

A Figura 3 apresenta o comportamento do fator $\Omega$ em função da porosidade para vários valores do número de Reynolds da partícula.

Verifica-se que o fator $\Omega$ aumenta com a diminuição da porosidade na faixa de porosidade apresentada na Figura $3(0,91$ a 0,99).

Figura 3 - $\mathrm{O}$ fator $\Omega$ em função da porosidade.

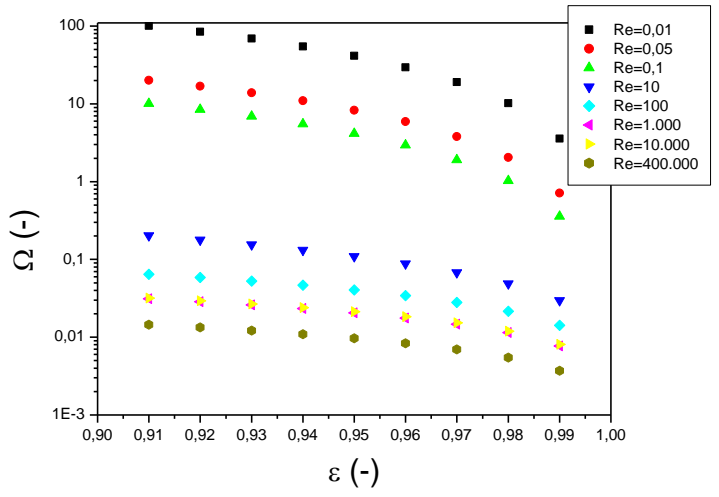

Fonte: Autor (2015).
O resultado encontrado de aumento do fator $\Omega$ com a diminuição da porosidade parece apresentar certa coerência quando se pensa na extrapolação do comportamento para porosidades bem menores, onde se sabe que o fator $\Omega$ apresenta um valor de 0,14 segundo Ergun (1952).

Em relação à parametrização dos regimes de escoamento, verifica-se na Figura 3 que a variação de $\Omega$ com a porosidade torna-se mais suave com o aumento do número de Reynolds da partícula.

O comportamento do fator $\Omega$ em função da porosidade para um determinado número de Reynolds pode ser descrito por uma equação com a seguinte forma:

$\Omega=a \cdot \exp \left[\mathrm{b} .(1-\varepsilon)^{\mathrm{c}}\right]$

A Figura 4 apresenta o comportamento do coeficiente de Burke-Plummer (B) em função da porosidade. Verifica-se um comportamento qualitativo de B em função da porosidade semelhante ao comportamento apresentado pelo fator $\Omega$.

Figura 4 - Constante de Burke-Plummer (B) em função da porosidade.

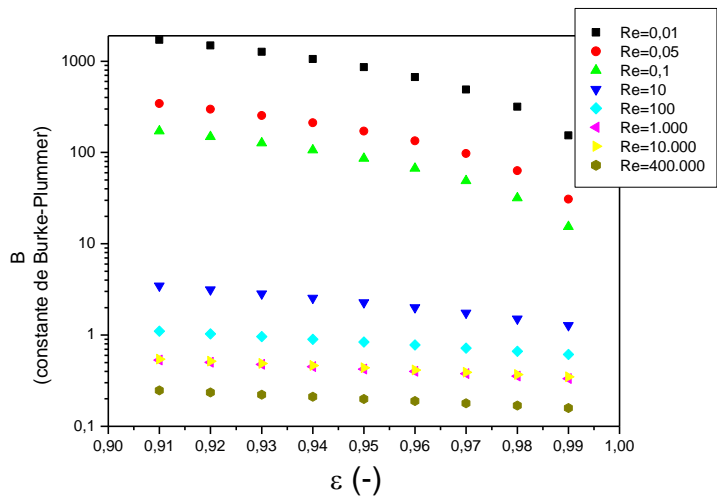

Fonte: Autor (2015).

\subsection{Comportamento Experimental do Fator $\Omega$ em função da Porosidade}

A fim de confrontar o comportamento teórico obtido para $\Omega$ em função da 
porosidade com o comportamento experimental, foram tomados os dados de $\varphi_{1} \mathrm{e}$ $\varphi_{2}$ apresentados por Massarani (2002) relativos aos experimentos realizados com cilindros equiláteros de $1,14 \mathrm{~mm}$ (Restini, 1977), esferas de vidro de $0,58 \mathrm{~mm}$ (Santana e Massarani, 1974) e areia de 0,2mm (Massarani e d'Ávila, 1974).

A partir dos valores obtidos de $\varphi_{1}$ e $\varphi_{2}$ calcularam-se os valores experimentais do fator $\Omega$ através da Equação 21. A Figura 5 apresenta os valores obtidos para $\Omega$.

Figura 5 - O fator $\Omega$ experimental em função da porosidade para diferentes partículas.

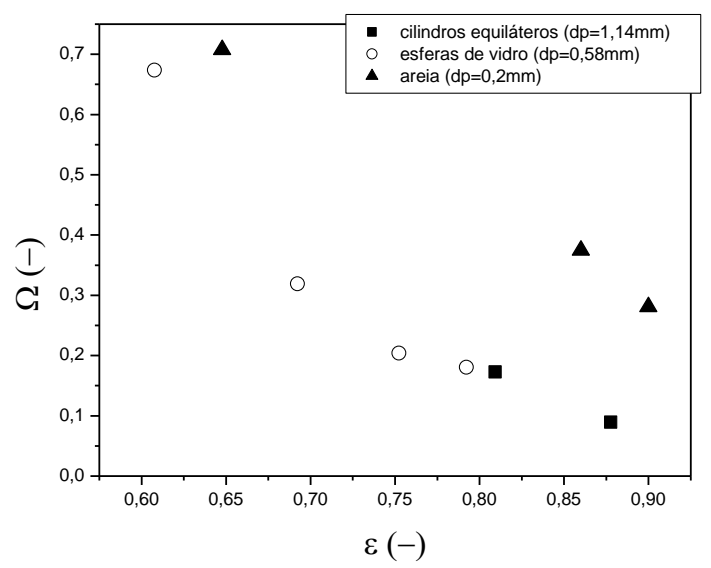

Fonte: Autor (2015).

Verifica-se que $\Omega$ diminui em função do aumento da porosidade para todas as partículas analisadas. Isso mostra que $o$ resultado teórico obtido (veja a Figura 3) parece ser coerente, pois também mantém a mesma tendência dos valores experimentais de $\Omega$ em função da porosidade apresentados na Figura 5, apesar destes serem relativos a porosidades inferiores a 0,9 , enquanto aqueles se referiam a porosidades tendendo à unidade $(\varepsilon \rightarrow 1)$.

\subsection{Equação para a Constante de Burke- Plummer em função do número de Reynolds e da Porosidade}

Devido ao fato da literatura apresentar correlações para a constante de BurkePlummer (B) em vez de correlações para o fator $\Omega$, foi realizado um estudo para a obtenção de uma equação para a constante de Burke-Plummer. Verificou-se que a equação para predizer os valores de B nos regimes de Stokes, de Newton e turbulento pode ser obtida da união das Equações 34 e 35 levando a:

$\mathrm{B}=\frac{\mathrm{a}}{\mathrm{Re}^{\mathrm{b}}} \cdot \exp \left[\mathrm{c} \cdot(1-\varepsilon)^{\mathrm{d}}\right]$

As constantes da Equação 36 foram tomadas como funções dos regimes de escoamento para a partícula isolada. A Tabela 1 apresenta os valores obtidos para as constantes para cada regime avaliado.

Tabela 1 - Constantes da Equação 36.

\begin{tabular}{ccccc}
\hline Regime & $\mathrm{a}$ & $\mathrm{b}$ & $\mathrm{c}$ & $\mathrm{d}$ \\
\hline Stokes & 0,00772 & 1,00 & 11,40 & 0,16 \\
Newton & 0,291 & $-0,012$ & 4,83 & 0,928 \\
Turbulento & 0,149 & 0 & 4,77 & 0,931 \\
Fonte: Autor & $(2015)$. & & &
\end{tabular}

As variâncias explicadas $\left(r^{2}\right)$ nos regimes de Stokes, de Newton e turbulento foram de 99,99\%, 99,93\% e $99,99 \%$ respectivamente.

Para o regime de transição na faixa de número de Reynolds da partícula isolada de $0,1<\operatorname{Re}<1$, a Equação 36 pode ser adotada com as constantes apresentadas na Tabela 2. A variância explicada foi de $99,95 \%$. Para as faixas de Reynolds de $1 \leq \operatorname{Re} \leq 10$ e $10<\operatorname{Re}<1.000$ os valores de $\mathrm{B}$ podem ser obtidos a partir de:

$\mathrm{B}=(\mathrm{a} \varepsilon+\mathrm{b}) \cdot \exp \left[\mathrm{c} \cdot \operatorname{Re}^{\mathrm{d}}\right]+\mathrm{e}$ 
respectivamente. Os valores obtidos para as constantes estão apresentados na Tabela 2.

Tabela 2 - Constantes das Equações 36 e 37 para o regime de transição.

\begin{tabular}{cccc}
\hline & $0,1<\operatorname{Re}<1$ & $1 \leq \operatorname{Re}<10$ & $10 \leq \operatorname{Re}<1.000$ \\
\hline $\mathrm{a}$ & 0,091 & $-0,0189$ & $-0,171$ \\
$\mathrm{~b}$ & 0,929 & 0,0190 & 0,175 \\
$\mathrm{c}$ & 9,167 & 9,32 & 6,89 \\
$\mathrm{~d}$ & 0,217 & $-0,102$ & $-0,137$ \\
$\mathrm{e}$ & - & 0,837 & 0,331
\end{tabular}

Fonte: Autor (2015).

A Figura 6 apresenta os valores preditos para B através das Equações 36 e 37 para porosidades acima de 0,9 e números de Reynolds relativos aos regimes de Stokes, de transição, de Newton e turbulento em função dos valores teóricos de B gerados a partir das Equações 16, 29 e 30.

É possível verificar através da Figura 6 uma boa concordância entre os valores observados e os valores preditos.

Figura 6 - Valores preditos de B em função dos valores observados.

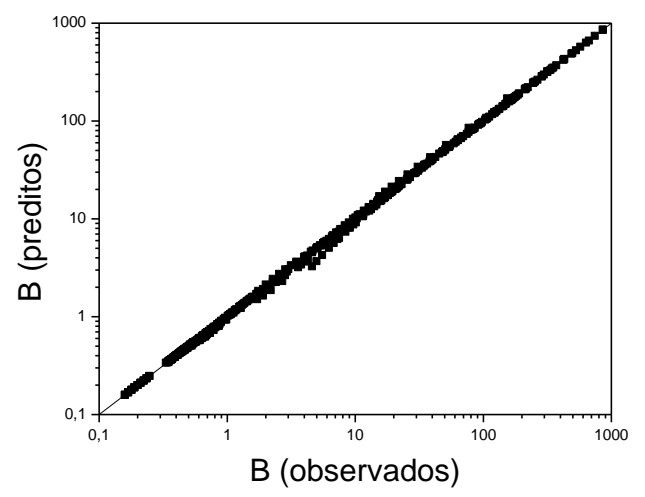

Fonte: Autor (2015).

3.5 Comparação dos Valores Obtidos para a Constante de Burke-Plummer neste Trabalho com os Obtidos a partir de Correlações da Literatura

Neste item apresenta-se uma comparação do coeficiente de Burke-Plummer obtido neste trabalho com o obtido por correlações de literatura.
Foram consideradas as seguintes correlações para a comparação:

(Hill et al., 2001) $\varepsilon . \operatorname{Re}>40$ e $\varepsilon>0,6$

$$
\mathrm{B}=0,6057 \cdot \varepsilon^{2}+1,908 \cdot(1-\varepsilon) \cdot \varepsilon^{2}+0,209 \cdot \varepsilon^{-3}
$$

(Beetstra et al., 2006) $\varepsilon . \operatorname{Re}<1.000$

$$
\mathrm{B}=\frac{0,31\left[\varepsilon^{-1}+3(1-\varepsilon) \varepsilon+8,4(\varepsilon \mathrm{Re})^{-0,343}\right]}{1+10^{3(1-\varepsilon)}(\varepsilon \operatorname{Re})^{2 \varepsilon-2,5}}
$$

(Plessis e Woudberg, 2008) para $\mathrm{C}_{\mathrm{D}}=1,9$

$$
\mathrm{B}=\frac{\varepsilon^{2} \cdot \mathrm{C}_{\mathrm{D}}}{2\left[1-(1-\varepsilon)^{2 / 3}\right]^{2}}
$$

A Figura 7 apresenta os valores da constante de Burke-Plummer obtidos em a partir das correlações representadas pelas Equações 39-41.

Figura 7 - Valores calculados de B por diferentes correlações em função da porosidade.

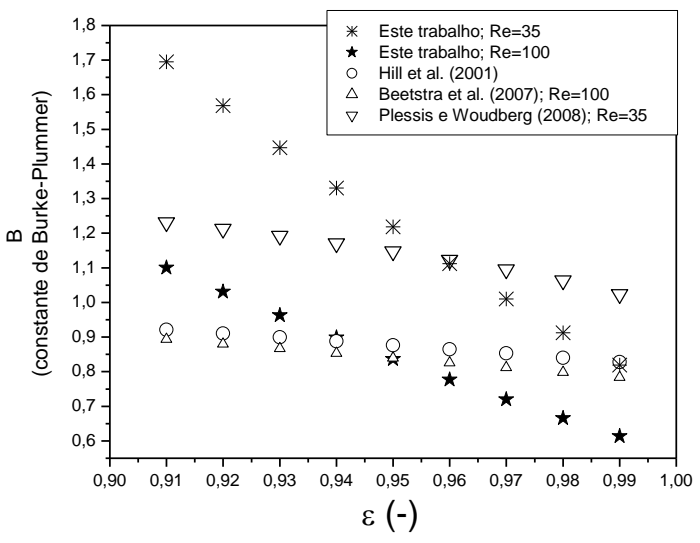

Fonte: Autor (2015).

É possível verificar que para porosidades de 0,91 a 0,94 as correlações de Hill et al. (2001) e Beetstra et al. (2007) 
subestimam os valores de $\mathrm{B}$ encontrados neste trabalho para $\mathrm{Re}=100$, enquanto que para porosidades acima de 0,95 os valores são superestimados. A correlação de Plessis e Woudberg (2008) subestima os valores de B encontrados neste trabalho para $\mathrm{Re}=35$ para porosidades abaixo de 0,96 e superestima para porosidades acima de 0,96 .

\section{CONCLUSÕES}

O objetivo do presente trabalho foi avaliar o comportamento teórico do fator $\Omega$ e da constante de Burke-Plummer (B) da equação de Blake-Kozeny-Carman em função da porosidade e do número de Reynolds da partícula em condições de porosidades elevadas.

Quanto à influência do número de Reynolds da partícula, verificou-se que $\Omega$ e B diminuem com o aumento do número de Reynolds da partícula.

Em relação à porosidade, o aumento da porosidade provoca uma queda em $\Omega$ e B na faixa de porosidade de 0,91 a 0,99 , o que foi verificado experimentalmente para porosidades em torno de 0,9 .

Foram propostas equações para o cálculo da constante de Burke-Plummer em função dos regimes de escoamento. As equações apresentaram variâncias explicadas $\left(\mathrm{r}^{2}\right)$ na faixa de 99,47 a $99,99 \%$.

\section{NOMENCLATURA}

A coeficiente de Blake-Kozeny-Carman

$A_{P} \quad$ área da partícula, $m^{2}$

B constante de Burke-Plummer

c fator c (Equação 8), (-)

$\mathrm{C}_{\mathrm{D}} \quad$ coeficiente de arraste (Equação 22), (-)

dp diâmetro da partícula, $m$

$\mathrm{k}$ permeabilidade, $\mathrm{m}^{2}$

$\mathrm{k}_{\mathrm{KOZ}} \quad$ constante de Kozeny, (-)

$\mathrm{L}$ comprimento, $\mathrm{m}$

$\begin{array}{ll}\mathrm{m} & \begin{array}{l}\text { força resistiva, } \mathrm{N} / \mathrm{m}^{3} \\ \text { pressão piezométrica, } \mathrm{Pa}\end{array} \\ \mathrm{P} & \begin{array}{l}\text { velocidade superficial do fluido, } \mathrm{m} / \mathrm{s} \\ \text { número de Reynolds da partícula }\end{array} \\ \mathrm{Re} & \begin{array}{l}\left(=\rho . d p . \mathrm{v}_{o} / \mu\right),(-) \\ \text { raio hidráulico, } \mathrm{m}\end{array} \\ \mathrm{R}_{\mathrm{h}} & \text { superfície específica molhada da matriz } \\ \mathrm{S}_{\mathrm{M}} & \begin{array}{l}\text { porosa, } \mathrm{m}^{2} / \mathrm{m}^{3} \\ \text { velocidade intersticial do fluido, } \mathrm{m} / \mathrm{s}\end{array} \\ \mathrm{u} & \begin{array}{l}\text { velocidade intersticial relativa fluido- } \\ \mathrm{U}\end{array} \\ & \begin{array}{l}\text { sólido, } \mathrm{m} / \mathrm{s} \\ \text { velocidade terminal da partícula, } \mathrm{m} / \mathrm{s}\end{array} \\ \mathrm{V}_{\infty} & \text { volume da partícula, } \mathrm{m}^{3}\end{array}$

\section{Letras Gregas}

$\beta \quad$ fator do escoamento em dutos, (-)

$\varepsilon \quad$ porosidade, $(-)$

$\phi \quad$ esfericidade, $(-)$

$\varphi_{1} \quad$ fator $\varphi_{1}$ (Equação 17), $\mathrm{m}^{-2}$

$\varphi_{2}$ fator $\varphi_{2}$ (Equação 18), $\mathrm{m}^{-1}$

$\mu \quad$ viscosidade dinâmica do fluido, $\mathrm{kg} /(\mathrm{m} . \mathrm{s})$

$\mu_{\text {ap }} \quad$ viscosidade aparente, $\mathrm{kg} /(\mathrm{m} . \mathrm{s})$

$\rho \quad$ massa específica do fluido, $\mathrm{kg} / \mathrm{m}^{3}$

$\rho_{\mathrm{S}} \quad$ massa específica do sólido, $\mathrm{kg} / \mathrm{m}^{3}$

$\Omega \quad$ fator $\Omega,(-)$

$\psi_{1}$ fator $\psi_{1}$ (Equação 25), (-)

$\psi_{2}$ fator $\psi_{2}$ (Equação 26), (-)

\section{REFERÊNCIAS}

BARNEA, E; MIZRAHI, J. A Generalized Approach to the Fluid Dynamics of Particulate Systems. Part 1. General Correlation for Fluidization and Sedimentation in Solid Multiparticle Systems. Chemical Engineering Journal, v.5, 171189, 1973.

BEETSTRA, R.; VAN DER HOEF, M.A.; KUIPERS, J.A.M. Drag Force from Lattice Boltzmann Simulations of Intermediate Reynolds Number Flow Past Mono- and Bidisperse Arrays of Spheres. A.I.Ch.E. Journal, v.53, 489-501, 2006. 
CARMAN, P.C. Fluid flow through granular beds. Transactions of the Institution of Chemical Engineers, v.15, 150-166, 1937.

COMITI, J.; RENAUD, M. A New Model for Determining Mean Structure Parameters of Fixed Beds from Pressure Drop Measurements: Application to Beds Packed with Parallelepipedal Particles. Chemical Engineering Science, v.44, 1539-1545, 1989.

D'ARCY, H. P. G. Les Fontaines Publiques de la Ville de Dijon. Paris: Victor Dalmont, 1856.

DEEN, N.G.; ANNALAND, M. V. S.; HOEF, M. A. V.; KUIPERS, J. A. M. Review of Discrete Particle Modeling of Fluidized Beds. Chemical Engineering Science, v.62, 28-44, 2007.

EISFELD, B.; SCHNITZLEIN, K. The Influence of Confining Walls on the Pressure Drop in Packed Beds. Chemical Engineering Science, v.56, 43214329, 2001.

ERGUN, S. Fluid Flow Through Packed Columns. Chem. Eng. Progress, v.48, 89-94, 1952.

FOUST, A. S.; WENZEL, L. A.; CLUMP, C. W.; MAUS, L.; ANDERSEN, L.B. Princípios das Operações Unitária. Rio de Janeiro: LTC, 1982.

HAPPEL, J.; BRENNER, H. Low Reynolds Number Hydrodynamics. London: Prentice-Hall, Inc., 1965.

HILL, R.J.; KOCH, D.L.; LADD, J.C. ModerateReynolds-Numbers Flows in Ordered and Random Arrays of Spheres. Journal of Fluid Mechanics, 448, 243-278, 2001.

MACDONALD, I. F.; EL-SAYED, M. S., MOW, K.; DULLIEN, F. A. L. Flow through Porous Media-the Ergun Equation Revisited. Industrial Engineering Chemical Fundamentals, v.18, 199208, 1979.

MASSARANI, G. Aspectos da Fluidodinâmica em Meios Porosos. Revista Brasileira de Engenharia, número especial, 96p., 1989.

MASSARANI, G. Fluidodinâmica em Sistemas Particulados. Rio de Janeiro: Editora E-papers, 2002.
MASSARANI, G.; d'ÁVILA, J. S. Transporte Vertical de Partículas Sólidas. In: Anais do II ENEMP, Rio Claro-SP, 1974, p.33-47.

MASSARANI, G.; SANTANA, C. C. Fluidização Homogênea: Caracterização Fluidodinâmica de Sistemas Particulados com Porosidade Elevada. In: Anais do Congresso Europeu de Fluidização, Las Palmas de Gran Canária, Espanha, 1994, p.83-91.

MOLERUS, O. A Coherent Representation of Pressure Drop in Fixed Beds and of Bed Expansion for Particulate Fluidized Beds. Chemical engineering Science, v.35, 1331-1340, 1980.

NEMECA, D.; LEVEC, J. Flow through Packed Bed Reactors: 1. Single-Phase Flow. Chemical Engineering Science, v.60, 6947-6957, 2005.

PLESSIS, J. P.; WOUDBERG, S. Pore-scale Derivation of the Ergun Equation to Enhance its Adaptability and Generalization. Chemical Engineering Science, v.63, 2576-2586, 2008.

PUNCOCHAR, M.; DRAHOS, J. Limits of Applicability of Capillary Model for Pressure Drop Correlation. Chemical Engineering Science, v.55, 3951-3954, 2000.

RESTINI, C.V. Transporte Vertical de Partículas Sólidas II: Análise Experimental. 1977. 57p. Dissertação (Mestrado em Engenharia Química), Universidade Federal do Rio de Janeiro, Rio de Janeiro, 1977.

RIBEIRO, A. M.; NETO, P.; PINHO, C. Mean Porosity and Pressure Drop Measurements in Packed Beds of Monosized Spheres: Side Wall Effects. International Review of Chemical Engineering, v. 2, 40-46, 2010.

ROSCOE, R. The Viscosity of Suspensions of Rigid Spheres. British Journal of Applied Physics, v.3, 267-269, 1952.

SANTANA, C. C.; MASSARANI, G. Força Resistiva no Escoamento em Meios Porosos de Alta Porosidade. Unimar, v. 1, 49-58, 1974.

ZHU, S.; PELTON, R. H.; COLLVER, K. Mechanistic Modelling of Fluid Permeation through Compressible Fiber Beds. Chemical Engineering Science, v.50, 3557-3572, 1995. 